\title{
Cloro, el ion olvidado y su relación con el suero fisiológico
}

The forgotten ion $\mathrm{Cl}$

\author{
ESTEBAN HUME $\vee^{1}$
}

\begin{abstract}
Chloride is the anion of highest concentration in the extracellular fluid, it contributes between 100 to $300 \mathrm{mosm} / \mathrm{L}$ to the tonicity of plasma[1], and its plasma concentration is frequently reported in the different services as part of the general evaluation of plasma electrolytes. However, chloremia is often not taken into account beyond its role in the acid-base calculation. In the last times, chloride has begun to take importance by diverse reports where it would be associated to dyschloremias and in particular the hyperchloremic acidosis with harmful effects in the organism[2]. On the other hand, we have normal saline, which is massively used worldwide[3]. With a composition characterized by supra-physiological levels of chloride[4], being the main cause of hyperchloremic acidosis[5]. The objective of this review is to show the importance of chloride in different systems of the body, its relationship with normal saline and in turn the relationship of this one with the potential deleterious effects of hyperchloremic acidosis.
\end{abstract}

\section{RESUMEN}

El cloro es el anión de mayor concentración en el líquido extracelular, aporta entre 100 a 300 mosm/L a la tonicidad del plasma[1], su concentración plasmática es frecuentemente informada en los distintos servicios como parte de la evaluación general de los electrolitos plasmáticos. Sin embargo, la cloremia muchas veces no es tomada en cuenta más allá de su rol en el cálculo ácido-base. En el último tiempo, el cloro ha comenzado a tomar importancia por diversos reportes donde se asociaría a las discloremias y en particular la acidosis hiperclorémica con efectos dañinos en el organismo[2]. Por otra parte, tenemos el suero fisiológico, el cual es masivamente usado a nivel mundial[3]. Con una composición caracte-
Key words: Chloride, normal saline, Hyperchloremic acidosis.

\section{Palabras clave:}

Cloro, Suero fisiológico, Acidosis hiperclorémica.

Interno VII Medicina, Facultad de Medicina, Universidad de Chile.

Fecha de recepción: 6 de marzo de 2018

Fecha de aceptación: 18 de marzo de 2018

\section{ORCID}

https://orcid.org/0000-0003-1369-442X.

\section{Correspondencia:}

Esteban Hume V.

estebanhume@gmail.com 
rizada por niveles supra fisiológicos de cloro[4], siendo el principal causante de la acidosis hiperclorémica[5]. El objetivo de esta revisión es mostrar la importancia que cumple el cloro en distintos sistemas del organismo, su relación con el suero fisiológico y a su vez la relación de este, con los potenciales efectos deletéreos de la acidosis hiperclorémica.

\section{Introducción}

I cloro es el anión extracelular más importante del organismo. Una persona promedio tiene 115 $\mathrm{g}$ de cloro en su organismo, proviniendo principalmente de la sal de la dieta. Con un peso molecular de 35,5 u, cuenta con diversas funciones entre las que se encuentran, balance ácido-base, movimiento de agua entre distintos compartimientos, actividad muscular y rol como modulador de la inmunidad, coagulación y de la función renal, entre otras. Es absorbido a lo largo de casi todo el intestino y su excreción es fundamentalmente por vía renal; del cloro que filtran los riñones se absorbe el 99,1\%, mayoritariamente en los túbulos proximales y el resto es eliminado por la orina[5],[6],[7],[8].

Por otro lado, en la práctica clínica la solución más usada es el suero fisiológico $(0,9 \%$ de $\mathrm{NaCl})$. Para hacerse una idea de lo masivo que es su uso, solamente en Reino Unido se infunden cerca de 10 millones de litros de este suero al año[3]. El cual trae niveles supra fisiológicos de cloro (154 mmol/L) y sodio (154 $\mathrm{mmol} / \mathrm{L})[4]$.

El suero fisiológico fue descrito por primera vez por el Dr Hamburguer a finales del siglo XIX[3], y ya desde principios del siglo $\mathrm{XX}$ datan los primeros reportes en contra de su uso indiscriminado debido a sus efectos potencialmente dañinos ${ }^{9}$, cabe considerar que siempre ha sido puesto en duda si el suero "fisiológico" debería considerarse fisiológico[10]. Con el transcurso de los años cada vez se va sumando más evidencia de sus potenciales efectos deletéreos[1],[2],[5],[11]. Un hecho interesante es que no se tiene claro cómo ni por qué alcanzó tanta popularidad, siendo que existían soluciones más fisiológicas en esa época[3]. Probablemente fue por motivos económicos, ya que, una bolsa de suero fisiológico cuesta US $\$ 0,86$ por litro mientras que el plasmalyte, un cristaloide balanceado, cuesta US\$ 1,62 por litro[4].

\section{Acidosis hiperclorémica}

Uno de los efectos más importantes que suce- den tras la administración de suero fisiológico es la generación de una acidosis hiperclorémica, la cual se puede ver en otras situaciones como cetoacidosis diabética y algunos tipos de acidosis tubular renal. Sin embargo, la etiología más común de este tipo particular de acidosis es su ocurrencia tras la administración de grandes cantidades de suero fisiológico[5],[12].

La fisiopatología de este fenómeno se logra explicar usando la teoría del balance ácido-base de Stewart, que cuenta con 3 variables independientes que serían las reguladoras del $\mathrm{pH}$, cuya variable principal es la carga neta de iones fuertes que existe en el organismo, de ahí nace el concepto de la diferencia de iones fuertes o SID (strong ion difference)[13], calculándose $\left(\mathrm{Na}^{+}+\mathrm{K}^{+}\right)-\left(\mathrm{Cl}^{-}+\right.$Lactato $)$. La SID normal del plasma es $40 \mathrm{meq} / \mathrm{L}$ y esta disminuye cuando los aniones aumentan desproporcionadamente en comparación a los cationes resultando en una disminución del pH y acidosis[10],[13]. Por lo que si aportamos una solución muy rica en cloro y sodio en relación 1:1 esta solución tendría una SID de 0, bajando la SID plasmática. Para compensar esta situación y mantener la electroneutralidad el organismo disocia iones hidrógeno, con lo que disminuye el $\mathrm{pH}[12]$. Las otras dos variables independientes reguladoras del $\mathrm{pH}$ de la teoría de Stewart son la concentración total de ácidos débiles no volátiles y la presión parcial de dióxido de carbono[10],[13]. Otro componente de la acidosis generada por la infusión de soluciones salinas vendría dado por el efecto dilucional del bicarbonato, asociada también a una disminución de la secreción de protones, secundaria a un aumento de la llegada de cloro a la mácula densa lo que lleva a una disminución de la secreción de renina y aldosterona, produciendo finalmente la disminución de excreción de protones a nivel renal, favoreciendo la acidosis[5],[14].

Es importante tener presente que la acidosis hiperclorémica se puede malinterpretar en una situación de reanimación como acidosis láctica secundaria a falta de volumen y seguir aportando más suero fisiológico lo que empeoraría el problema[15]. Por ejemplo, en situaciones de trauma mayor donde se requiere reponer grandes cantidades de volumen, se ha observado que la hipercloremia se asocia a una 
mayor mortalidad[16]. Por todo esto es importante calcular la SID cuando estemos en presencia de una acidosis para descartar que pueda estar involucrado un componente como el cloro[4].

\section{Soluciones balanceadas}

Como contraparte al suero fisiológico, tenemos, las soluciones balanceadas, las cuales se caracterizan por tener una composición similar al líquido extracelular, con concentraciones de cloro menor a los 110 meq/L, entre ella encontramos el Ringer lactato, plasmalyte A, plasmalyte 148 entre otras, con algunas leves diferencias en su composición que en ciertas situaciones nos pueden hacer preferir una sobre otra, sin embargo, no es el objetivo dicho análisis en esta revisión[14].

En las soluciones balanceadas la SID es similar al plasmático, por lo que el pH se ve afectado en menor medida, lo cual se ha visto clínicamente en distintos trabajos, donde las soluciones balanceadas generan menos alteraciones electrolíticas y ácido-base en comparación con soluciones no balanceadas como el suero fisiológico[4],[15],[17],[18]. Las soluciones balanceadas contienen aniones orgánicos que actúan como buffers, y también contienen cationes, que, aunque no tienen un rol fisiológico claro en el balance ácido básico podrían influir en este[19].

\section{Cloro y sistema inmune}

En el último tiempo ha comenzado a tomar cada vez más importancia la relación del cloro con el sistema inmune, este ion es un elemento esencial en la inmunidad innata siendo sustrato fundamental para la generación de potentes microbiocidas como el ácido hipocloroso[20]. Estudios in vivo muestran un aumento de las citoquinas en el plasma tras la inducción de una acidosis hiperclorémica[8]. A nivel de la inmunidad celular, el cloro modula distintos tipos celulares como las células T CD4+, macrófagos y células natural killer. Se piensa que el mecanismo de inducción es mediante estrés osmótico, por señales activadas a causa de la tonicidad[21]. También en la acidosis hiperclorémica se ha observado un aumento de marcadores inflamatorios, asociado a la alteración en generación de especies reactivas del oxígeno, siendo este efecto más marcado en la acidosis hiperclorémica que en otro tipo de acidosis como por ejemplo la acidosis láctica[22]. Interesante es lo que sucede en la fibrosis quística donde la alteración del canal de cloro CFTR, finalmente termina afectando la inmunidad tanto innata como adaptativa[23]. También el uso de suero fisiológico se ha asociado a mayor riesgo de tener infecciones post operatorias comparado con soluciones balanceadas[11],[24]. Con todos estos antecedes se puede decir que el cloro tendría una importante función tanto en la inmunidad innata como adaptativa.

\section{Cloro y coagulación}

Un tema importante en relación con el cloro y en particular al suero fisiológico, es si de alguna manera altera la coagulación, hecho importante dada la gran cantidad de volumen que se puede llegar a perder en el acto quirúrgico, o en la reanimación de un politrauma, situaciones donde idealmente se requieren soluciones que no alteren o alteren lo menos posible la coagulación. Estudios in vitro han mostrado que soluciones hiperclorémicas en relación con el plasma producen estados de hipocoagulabilidad en comparación con soluciones normoclorémicas, sin embargo, esto comienza a ocurrir a altas concentraciones de cloro, con niveles que son difícilmente logrables en la clínica[7]. Lo cuales concordante con el estudio de Song JW et al[6], donde se estudió el efecto en la coagulación del suero fisiológico, y se comparó con plasmalyte, observándose en ambas, un estado de hipocoagulabilidad, aunque sin diferencias estadísticamente significativas entre ambas. La causa de esta hipocoagulabilidad probablemente sea por mecanismos de hemodilución. Un reciente metaanálisis tampoco muestra mayores pérdidas sanguíneas intraoperatorias ni mayor necesidad de volumen con el uso de suero fisiológico comparado con soluciones balanceadas[18]. Sin embargo, en la literatura se describe un mayor riesgo de sangrado y necesidad de trasfusiones con suero fisiológico en comparación a soluciones balanceadas[11],[19],[24].

\section{Cloro y sistema digestivo}

El cloro tiene una importante relación con el sistema digestivo, este anión es absorbido a lo largo de casi todo el intestino. En el jugo gástrico se secreta cloro como ácido clorhídrico $(\mathrm{HCl})$, generándose alrededor de $8 \mathrm{~L}$ de este al día, el cual sirve para activar distintas enzimas proteolíticas que ayudarán con la digestión. Otro hecho importante es que el movimiento de agua hacia el intestino no puede ser llevado a cabo mediante transporte activo, por lo que el principal mecanismo para realizar este desplazamiento es la fuerza osmóti- 
ca, siendo el cloro el principal generador del gradiente osmótico que contribuye a este proceso[5].

En un estudio in vivo se observa que tras la infusión de suero fisiológico se generan alteraciones de la contractibilidad intestinal y edema[10]. Es interesante como el suero fisiológico en comparación con cristaloides balanceados, produce un empeoramiento en la oxigenación de la mucosa gástrica lo que se interpreta como un empeoramiento en su perfusión[17]. También se ha visto que la hipercloremia genera una vasoconstricción a nivel de la arteria esplácnica, de la misma forma que lo hace en las arteriolas renales[12],[16].

\section{Cloro y función renal}

A mediados del siglo XX Wilcox et al comenzó a estudiar el efecto que tenían distintas sustancias hipertónicas en la hemodinamia renal. Estudiaron en modelos experimentales con riñones de perro, observándose que las soluciones con concentraciones elevadas en cloro disminuyen significativamente la velocidad de filtración glomerular, secundaria a una vasoconstricción de la arteria renal. Otro hecho de interés es que, en situaciones de depleción de sal este efecto de disminución de la velocidad de filtración glomerular, flujo renal y perfusión renal aumentaba al doble[25]. En un modelo in vivo en perros se observa que la hipocloremia no disminuye la VFG, pero si aumenta la excreción de sodio y de potasio y se genera una alcalosis metabólica que una vez establecida no revierte con soluciones ricas en cloro[26]. Posteriormente, se comenzaron a realizar estudios en humanos donde se comparaba la infusión de suero fisiológico con soluciones balanceadas como el plasmalyte 148. Lo que se observó fue un aumento inicial y luego una disminución significativa del flujo de la arteria renal post infusión de $2 \mathrm{~L}$ suero fisiológico, lo que no ocurrió tras la administración del cristaloide balanceado[27]. Estudios clínicos son concordantes con esta observación donde tras la administración de suero fisiológico se genera una disminución del débito urinario[6],[15].

Con todos estos antecedentes se realizó el estudio SPLIT, un estudio clínico prospectivo, cuyo objetivo era determinar las complicaciones que se generaban tras la administración de suero fisiológico comparada con una solución balanceada en pacientes admitidos en una unidad de cuidados intensivos. Contó con 2.278 pacientes post operados, donde se observó que no existía diferencia significativa entre el uso de suero fisiológico y una solución balanceada en el desarrollo de daño renal agudo (AKI), ni diferencias de mortalidad a 90 días, ni aumento de necesidad de terapias de reemplazo renal. Se ha criticado este estudio por la falta de datos al exponer las características entre los 2 grupos, no describen el total de solución que administraron, además que previamente ya se había pasado soluciones preferentemente con algún buffer y era una población con baja incidencia de AKI[28]. Posteriormente, se estudió la relación entre hipercloremia y desarrollo de AKI a las 72 horas post ingreso en una unidad de cuidados intensivos, en donde no se observó relación entre hipercloremia con el desarrollo de AKI[29], sin embargo, este estudio incluía gente severamente enferma, y no se explicita con qué solución se rehidrataba, tampoco muestra la cantidad ni el tipo de solución que se entregó previamente, y no se midió el débito de orina en el tiempo; sólo se midió cloro al inicio y a las 72 horas.

Hay ciertas situaciones donde existe elevado riesgo de hiperkalemia como es el caso de los trasplantes renales[30]. Por esta razón la recomendación era que durante el trasplante se usara suero fisiológico por ser una solución libre de potasio; sin embargo, distintos estudios han mostrado que, en comparación a soluciones balanceadas el suero fisiológico produce más hiperkalemia, debido a la acidosis hiperclorémica que genera, la que estimula el intercambio de hidrogeniones por potasio al extracelular[30],[31]. A opinión del autor esta situación es extrapolable a otros escenarios con alto riesgo de hiperkalemia como sería el caso de la insuficiencia renal crónica, sin embargo, falta literatura al respecto.

\section{Cloro y sepsis}

La acidosis hiperclorémica es común en los pacientes críticos y en general es iatrogénica[8], ya que durante la resucitación en pacientes en shock sépticos la fluidoterapia es un pilar fundamental en el tratamiento. El fluido más usado es el suero fisiológico, sin embargo, el último tiempo han existido reportes donde en comparación con soluciones balanceadas tendría peores outcomes[19]. Una serie de estudios, incluyendo metaanálisis mostraba que, durante la resucitación en pacientes sépticos, el grupo que recibía soluciones balanceadas tenía asociada una menor mortalidad en comparación a los que recibían suero fisiológico[28],[32], encontrándose que el cloro tiene una asociación independiente del volumen infundido con la mortalidad[33]. Por otra parte, también se ha asociado una relación entre hipocloremia y muerte en pacientes en shock séptico o sepsis severa y que al 
aumentar la cloremia disminuiría esta asociación significativamente[34]. Pese a que la evidencia se basa en estudios observacionales, hasta el momento la evidencia indica que se deberían preferir soluciones balanceadas sobre el suero fisiológico en la reanimación en sepsis[19].

\section{Hipocloremia}

Al hablar de discloremias, la que se ha llevado la mayoría de la atención han sido las hipercloremias, sin embargo, hay muchas situaciones clínicas que pueden llevar a una hipocloremia, teniendo causas gastrointestinales, renales o por mecanismos de hemodilución, siendo la etiología más frecuente, la secundaria a vómitos. Esto es relevante ya que el cloro se relaciona inversamente con el bicarbonato, por lo que cuando existe un balance negativo de cloro, el bicarbonato aumenta proporcionalmente, generándose una alcalosis metabólica[5].

Existe poca bibliografía y estudios sobre la hipocloremia y sus eventuales efectos deletéreos a nivel clínico. A mediados del siglo XX, el método para estudiar los efectos de la hipocloremia era mediante el retiro de jugo gástrico del organismo en estudio[35] Tani et al observó que la hipocloremia se relaciona con un mayor SID y una alcalosis lo que es concordante con la teoría de Stewart[13],[36], también se ha observado que no empeora la función renal[37].

En cuanto a la clínica, los efectos más estudiados han sido sobre patología cardiovascular. Se realizó un estudio donde se vio que la hipocloremia se asociaba con un aumento de la mortalidad en pacientes con accidente cerebrovascular agudo[38], y también previamente se había observado que era una variable independiente en falla cardiaca. Uno de los mecanismos de porque aumentaría los eventos cardiovasculares seria que a menor cloremia hay un mayor anión GAP y mayores niveles de renina, asociándose a mayor presión que llevaría a aumentar la mortali- dad y peores outcomes[39].

\section{Conclusiones}

A lo largo de esta revisión se puede observar la importancia del cloro en distintos sistemas, y cómo su alteración puede modificar su funcionamiento. Por lo anterior, es importante no pasar por alto una discloremia, y siempre buscar una causa subyacente, dado que estudios tanto in vitro como in vivo, y ensayos clínicos nos muestran su potencial efecto deletéreo en los distintos sistemas.

Desde el punto de vista fisiopatológico quedan pocas dudas sobre los efectos nocivos del suero fisiológico, dados principalmente por la acidosis hiperclorémica que genera. Sin embargo, a nivel clínico la evidencia es contradictoria en algunos temas, por ejemplo, en su capacidad de generar mayor riesgo de sagrado o mayor necesidad de transfusión. Tampoco hay certeza a nivel clínico si realmente aumenta la incidencia de fallas renales aguda o la necesidad de terapias de reemplazo renal. Estos resultados contradictorios posiblemente se vayan dilucidando en el tiempo, con una mayor cantidad estudios prospectivos, dado que la evidencia hasta el momento se basa en su mayoría en estudios retrospectivos.

En donde existe algo más de certeza es en el territorio de la sepsis, en especial en el shock séptico, donde la evidencia continúa mostrando la superioridad en reanimación con soluciones balanceadas en comparación al suero fisiológico.

A pesar de que desde un punto de vista fisiológico no se debería usar suero fisiológico, por las razones ya expuestas, clínicamente aún falta evidencia para no recomendar su uso. Es importante reconocer que en ciertas situaciones su uso puede ser necesario, y en caso de no contar con otras alternativas, puede ser una buena opción. Sin embargo, recordar siempre tener una mirada crítica sobre qué tipo de soluciones estamos aportando al paciente y en qué contexto.

\section{Referencias}

1. Koch SM, Taylor RW. Chloride ion in intensive care medicine. Crit Care Med 1992;20(2):227-40. http:// dx.doi.org/10.1097/00003246199202000-00012

2. Bandak G, Kashani KB. Chloride in intensive care units: a key electrolyte. F1000Res. 2017 Nov 1;6:1930. doi: 10.12688/ f1000research.11401.1

3. Awad S, Allison SP, Lobo DN. The history of $0.9 \%$ saline. Clin Nutr. 2008 Apr;27(2):179-88. i:10.1016/j.cInu.2008.01.008.

4. Barker ME. 0.9\% saline indu- ced hyperchloremic acidosis. J Trauma Nurs. 2015 MarApr;22(2):111-6. doi: 10.1097/ JTN.0000000000000115.

5. Kenrick Berend, Leonard Hendrik van Hulsteijn, Rijk O.B. Gans. Chloride: The queen of electrolytes?. Eur J Intern Med. 2012 Apr;23(3):203-11. doi: 
10.1016/j.ejim.2011.11.013.

6. Song JW, Shim JK, Kim NY, Jang J, Kwak YL. The effect of $0.9 \%$ saline versus plasmalyte on coagulation in patients undergoing lumbar spinal surgery; a randomized controlled trial. Int J Surg. 2015 Aug;20:128-34. doi:10.1016/j.ijsu.2015.06.065.

7. Roche AM, James MF, BennettGuerrero E, Mythen MG. A head-to-head comparison of the in vitro coagulation effects of saline-based and balanced electrolyte crystalloid and colloid intravenous fluids. Anesth Analg. 2006 Apr;102(4):1274-9.

8. Kellum JA, Song M, Almasri E. Hyperchloremic acidosis increases circulating inflammatory molecules in experimental sepsis. Chest. 2006 Oct;130(4):962-7.

9. Evevans $\mathrm{GH}$. The Abuse of normal salt solution. JAMA. 1911;LVII(27):2126-2127. doi:10 .1001/a.1911.04260120316010،

10. Li H, Sun SR, Yap JQ, Chen JH, Qian Q. 0.9\% saline is neither normal nor physiological. J Zhejiang Univ Sci B. 2016 Mar;17(3):181-7. doi: 10.1631/ jzus.B1500201.

11. Maristella Santi, Sebastiano A. G. Lava, Pietro Camozzi, et al. The great fluid debate: saline or socalled "balanced" salt solutions? Ital J Pediatr. 2015 Jun 25;41:47. doi: 10.1186/s13052-015-01542.

12. Burdett E, Roche AM, Mythen MG. Hyperchloremic Acidosis: Pathophysiology and Clinical Impact. Transfusion Alternatives in Transfusion Medicine, October 2003 5: 424-430. doi:10.1111/ j.1778-428X.2003.tb00184.x

13. Stewart PA. Modern quantitative acid-base chemistry. Can J Physiol Pharmacol. 1983 Dec;61(12):1444-61.

14. El Gkotmi N, Kosmeri C, Filippatos TD, Elisaf MS. Use of intravenous fluids/solutions: a narrative review. Curr Med Res Opin. 2017 Mar;33(3):459-471. doi: 1080/03007995.2016.1261819.

15. Williams EL, Hildebrand KL, McCormick SA, Bedel MJ. The effect of intravenous lactated Ringer's solution versus $0.9 \%$ sodium chloride solution on serum osmolality in human volunteers. Anesth Analg. 1999 May;88(5):999-1003.

16. Lee JY, Hong $\mathrm{TH}$, Lee KW, Jung MJ, Lee JG, Lee SH. Hyperchloremia is associated with 30-day mortality in major trauma patients: a retrospective observational study. Scand J Trauma Resusc Emerg Med. 2016 Oct 4;24(1):117.

17. Wilkes NJ, Woolf R, Mutch M, Mallett SV, Peachey T, Stephens $R$, Mythen MG. The effects of balanced versus saline-based hetastarch and crystalloid solutions on acid-base and electrolyte status and gastric mucosal perfusion in elderly surgical patients. Anesth Analg. 2001 Oct;93(4):811-6.

18. Huang L, Zhou X, Yu H. Balanced crystalloids vs $0.9 \%$ saline for adult patients undergoing non-renal surgery: A meta-analysis. Int J Surg. 2018 Mar;51:1-9. doi: 10.1016/j.ijsu.2018.01.003.

19. Loflin R, Winters ME. Fluid Resuscitation in Severe Sepsis. Emerg Med Clin North Am. 2017 Feb;35(1):59-74. doi: 10.1016/j. emc.2016.08.001

20. Wang G, Nauseef WM. Salt, chloride, bleach, and innate host defense. Journal of Leukocyte Biology, 2015 Jun 98: 163-172. doi:10.1189/jlb.4RU0315-109R.

21. Jeong $D, K i m ~ H Y, C h u n g ~ D H$. Sodium chloride inhibits IFN- $\gamma$, but not IL-4, production by invariant NKT cells. J Leukoc Biol. 2018 Jan;103(1):99-106. doi: 10.1002/JLB.3A0217-076R

22. John A Kellum, Mingchen Song, and Jinyou Li Science re- view: Extracellular acidosis and the immune response: clinical and physiologic implications Crit Care. 2004 Jun; 8(5): 331-336. doi: $10.1186 /$ cc2900

23. Döring G, Gulbins E. Cystic fibrosis and innate immunity: how chloride channel mutations provoke lung disease. Cell Microbiol. 2009 Feb;11(2):20816. doi: 10.1111/j.14625822.2008.01271.x.

24. Shaw AD, Bagshaw SM, Goldstein SL, et all. Surgery. Major complications, mortality, and resource utilization after open abdominal surgery: $0.9 \%$ saline compared to Plasma-Lyte. Ann Surg. 2012 May;255(5):821-9. doi: 10.1097/ SLA.0b013e31825074f5.

25. Wilcox CS. Regulation of renal blood flow by plasma chloride. J Clin Invest. 1983 Mar;71(3):726-

26. Toussaint $C$, Telerman M, Vereerstraeten $P$. Effects of acute hypochloremia on glomerular filtration rate and electrolyte excretion in the dog. Experientia. 1958 Nov 15;14(11):417-9.

27. Chowdhury AH, Cox EF, Francis ST, Lobo DN. A randomized, controlled, double-blind crossover study on the effects of 2-L infusions of $0.9 \%$ saline and plasma-lyte $₫ 148$ on renal blood flow velocity and renal cortical tissue perfusion in healthy volunteers. Ann Surg. 2012 Jul;256(1):18-24. doi: 10.1097/ SLA.0b013e318256be72.

28. Young P, Bailey M, Beasley $R$, et al. Effect of a Buffered Crystalloid Solution vs Saline on Acute Kidney Injury Among Patients in the Intensive Care Unit: The SPLIT Randomized Clinical Trial. JAMA. 2015 Oct 27;314(16):1701-10. doi: 10.1001/jama.2015.12334.

29. Lenar Yessayan, Javier A. Neyra, Fabrizio Canepa-Escaro, George Vásquez-Ríos,Michael Heung, 
Jerry Yee, y the Acute Kidney Injury in Critical Illness Study Group. Effect of hyperchloremia on acute kidney injury in critically ill septic patients: a retrospective cohort study BMC Nephrol. 2017; 18: 346. Doi. 10.1186/ s12882-017-0750-z

30. Weinberg L, Harris L, Bellomo R, lerino FL et al. Effects of intraoperative and early postoperative normal saline or Plasma-Lyte $148 \AA$ on hyperkalaemia in deceased donor renal transplantation: a double-blind randomized trial. Br J Anaesth. 2017 Oct 1;119(4):606-615. doi: 10.1093/ bja/aex163.

31. O'Malley CM, Frumento RJ, Hardy MA, et al. A randomized, double-blind comparison of lactated Ringer's solution and $0.9 \% \mathrm{NaCl}$ during renal transplantation. Anesth Analg. 2005 May;100(5):1518-24.

32. Rochwerg B, Alhazzani W, Sindi $A$, et al. Fluids in Sepsis and
Septic Shock Group. Fluid resuscitation in sepsis: a systematic review and network meta-analysis. Ann Intern Med. 2014 Sep 2;161(5):347-55. doi: 10.7326/ M14-0178.

33. Shaw AD, Raghunathan K, Peyerl FW, Munson SH, Paluszkiewicz SM, Schermer CR. Association between intravenous chloride load during resuscitation and in-hospital mortality among patients with SIRS. Intensive Care Med. 2014 Dec;40(12):1897905. doi: 10.1007/s00134-0143505-3.

34. Hyung Jung Oh, Seung Jun Kim, Yong Chan Kim, et al. An increased chloride level in hypochloremia is associated with decreased mortality in patients with severe sepsis or septic shock. Sci Rep. 2017 nov; 7: 15883. 10.1038/ s41598-017-16238-z.

35. Irving M Ariel. The Effects of Acute Hypochloremia on the Distribution of Body Fluid and
Composition of Tissue Electrolytes in Man. Ann Surg. 1954 Aug; 140(2): 150-163.

36. Tani M, Morimatsu $H$, Takatsu F, Morita K. The incidence and prognostic value of hypochloremia in critically ill patients. ScientificWorldJournal. 2012;2012:474185. doi: $10.1100 / 2012 / 474185$.

37. Joseph B. Kirsner, Walter Lincoln Palmer, Kathryn Knowlton. Studies on experimental and clinical hypochloremia in man. J Clin Invest. 1943 Jan; 22(1): 95-102. doi: 10.1172/JCI101373.

38. Bei HZ, You SJ, Zheng D, et al. Prognostic role of hypochloremia in acute ischemic stroke patients. Acta Neurol Scand. 2017 Dec;136(6):672-679. doi: 10.1111/ane.12785.

39. Radulovic B, Potocnjak I, Dokoza Terešak S, et al. Hypochloraemia as a predictor of developing hyponatraemia and poor outcome81 